\title{
Interpretation of the q-Deformed 1-D Quantum Harmonic Oscillator
}

\author{
A. S. Mahmood and M. A. Z. Habeeb \\ Department of Physics, College of Science, Al-Nahrain University, Baghdad, Iraq.
}

\begin{abstract}
The interpretation of the q-deformed 1-D quantum harmonic oscillator is investigated for two definitions of q-deformation. This investigation is achieved by using Zaslavskii's method to obtain the Heisenberg equations of motion (quantum Liouville equations) in the undeformed phase space. These quantum Liouville equations exhibit a non-commutative geometry produce from the existence of the dilatation operator which is inherent in the q-deformation process. The classical limits of these equations are obtained by applying a special classical limiting condition to produce the classical Liouville equations of the q-deformed oscillator. These classical Liouville equations are solved by using the method of characteristics in order to obtain the classical probability distribution functions for this system. The 2-D and 3-D behaviors of these functions were then investigated using a computer visualization method. The results of the mathematical derivations together with the computer visualization method show that the classical limit of the quantum Liouville equations for the q-deformed 1-D quantum harmonic oscillator are statistical in nature where the nonlinearity parameter for the q-deformed oscillator is connected with $\mathrm{h}$. This result conforms to that obtained by Ghosh et al. for the undeformed 1-D quantum harmonic oscillator.
\end{abstract}

The obtained classical probability distribution functions exhibit whorl shapes that evolve with time in phase space that are similar to the shapes obtained for the 1-D classical q-deformed oscillator. These whorl shapes in phase space are similar to those introduced by Milburn for the 1-D classical anharmonic oscillator. This similarity results from the fact that the anharmonicity itself represents a kind of deformation with a frequency that is a function of amplitude.

Keywords: quantum q-deformed oscillator, classical limit, classical Liouville equation, method of characteristics, classical probability distribution function.

\section{Introduction}

There have been many attempts performed to reveal the meaning and the interpretation of q-deformation [1-9]. In this context, the q-deformed quantum harmonic oscillator was used as a good model example. In 1991, Buzek [1] evaluated the time-evolution of the mean values of the q-position and the q-momentum for the q-oscillator in order to obtain the periodic classical behavior. In this treatment, the nonperiodic behavior of this oscillator was interpreted as the interaction of the quantum oscillator with another system. Shabanov [2] studied also the meaning and the interpretation of the same oscillator used by Buzek [1] but in a different manner. In 1992, Shabanov [2] obtained the q-deformed variables by using the standard Heisenberg commutation relations, and defined the qdeformation parameter, $q$, to be a function of the Planck constant and some dimensional parameter, $\ell_{q}$ where $q=e^{-\hbar / \ell_{q}^{2} \omega}$. To interpret this oscillator, 
he applied the classical limit $\hbar \rightarrow 0$, $q \rightarrow 1$ for the canonical variables to arrive at the classical theory. The second attempt by Shabanov [3] was more rigorous than the first one, where he introduced in 1993 the path integral in his approach. Hence, the classical theory was obtained by applying the semi classical approximation. It turns out that the q-oscillator can be interpreted as a particle with a friction force acting on the particle, and this force is proportional to the particle velocity. Man'ko et al. [4] studied both the quantum and classical q-oscillator via the Dirac dequantization method to construct the classical q-oscillator from the corresponding quantum q-oscillator and interpreted the q-oscillator as a classical non-linear oscillator with a special type of nonlinearity, where the frequency of the oscillator is a function of the energy which is a constant of the motion. Man'ko has also dealt with the f-oscillator in the same context in 1997 and 1998 [5, 6].

Furthermore Gruver [7] studied the dynamical properties of the q-deformed oscillator and found that this oscillator can be interpreted as an anharmonic oscillator with a q-deformation parameter which can be interpreted as a measure of anharmonicity. Another attempt to interpret the q-deformation can be found in the work of Batouli and El Baz [8] who studied the q-deformation for the quantum harmonic oscillator in a way similar to that of Buzek [1], but with some modifications. These modifications led to a different interpretation for the q-deformation where the q-deformed quantum harmonic oscillator can be considered as the quantum version of a classical forced oscillator with a modified q-dependent frequency, such that in the limit $q \rightarrow 1$, the driving force disappears. From another point of view, the q-deformation can be interpreted in terms of the noncommutative quantum mechanics. In this context, Lavagno et al. [9] investigated the meaning of q-deformation by applying non-commutative q-calculus.
Then, they were able to obtain the generalized q-classical theory in terms of the q-deformed Poisson bracket [9].

Eftekharzadeh et al. and Benatti et al. [10-12] also investigated the interpretation of the non-commutative quantum mechanics by applying the classical limit. In spite of all the attempts to interpret q-deformation mentioned above, there is still a problem facing the understanding of the physics behind this kind of deformation. The present paper is an attempt to investigate the nature of $\mathrm{q}$ deformation for the q-deformed quantum oscillator by using Zaslavskii's method [13] to obtain the Heisenberg equation of motion (quantum Liouville equation), then approach the classical limit to recover the classical Liouville equation of the qdeformed oscillator.

The rest of the paper is organized as follows. First, the q-deformed quantum harmonic oscillator is discussed where its Hamiltonian is introduced for both types of q-deformation. Then, the equations of motion and the Liouville equations are derived by using Zaslavskii's method [13]. The solutions for these Liouville equations are obtained by using the method of characteristics, then used to simulate the behavior in two and three dimensions and finally the conclusions are presented.

\section{q-Deformed 1-D Quantum Harmonic Oscillator}

In general, there are different versions of the q-deformed quantum harmonic oscillator according to the $\mathrm{q}$-commutator that is adopted for each version as well as to the definitions of the bosonic operators that satisfy these q-commutators [14-19]. An example of the q-deformed quantum oscillator is given in ref. [15] in which Biedenharn introduced the following q-commutator:

$\left[\hat{a}_{q}, \hat{a}_{q}^{\dagger}\right]_{q}=\hat{a}_{q} \hat{a}_{q}^{\dagger}-q^{\mp 1} \hat{a}_{q}^{\dagger} \hat{a}_{q}=q^{ \pm \hat{N}}$

However, according to Man'ko [4], the q-deformed oscillator represents a special 
type of nonlinearity where the frequency of the oscillator depends on the energy of the oscillator (i.e., $|\alpha|^{2}$ ). In this context, an f-deformed oscillator, which is a generalization of the q-oscillator, was introduced by Man'ko [5]. The realization of the f-deformed boson operators $\hat{a}_{f}$ and $\hat{a}_{f}^{\dagger}$ in terms of the undeformed boson operators $\hat{a}$ and $\hat{a}^{\dagger}$ was achieved via the transformation [4-6]:

$$
\left.\begin{array}{l}
\hat{a}_{f}=f(\hat{N}) \hat{a}=f(\hat{N}+1) \hat{a} \\
\hat{a}_{f}^{\dagger}=\hat{a}^{\dagger} f(\hat{N})=\hat{a}^{\dagger} f(\hat{N}+1)
\end{array}\right\}
$$

where $f(\hat{N})$ represents a non-negative real operator-valued function of the number operator. It should be noted that the subscript "f" used here refers to the "fdeformation" case. Also, whenever a qdeformation process is used instead of the f-deformation process, then the subscript " $\mathrm{f}$ " is interchanged by " $\mathrm{q}$ " and vice versa. The transformation from the f-deformed oscillator to the q-deformed oscillator or to the undeformed oscillator involves substituting specific values for the function $f(\hat{N})$ in the transformation of eqn. (2) in the form [4-6]:

$$
\begin{aligned}
& f(\hat{N})= \\
& \left\{\begin{array}{cc}
1 & \text { for undeformed oscillator } \\
\sqrt{\frac{[\hat{N}]_{q}}{\hat{N}}} & \text { for q-deformed oscillator } \\
\text { Otherwise } & \text { for f-deformed oscillator }
\end{array}\right.
\end{aligned}
$$

where,

$$
\begin{aligned}
& {[\hat{N}]_{q}=\frac{\sinh (\lambda \hat{N})}{\sinh (\lambda)}} \\
& \text { for }[\hat{N}]_{q}=\frac{q^{\hat{N}}-q^{-\hat{N}}}{q-q^{-1}}
\end{aligned}
$$

and,

$$
\begin{aligned}
& {[\hat{N}]_{q}=\frac{e^{\lambda \hat{N}}-1}{e^{\lambda}-1}} \\
& \text { for }[\hat{N}]_{q}=\frac{q^{\hat{N}}-1}{q-1}
\end{aligned}
$$

Furthermore, the Hamiltonian operators of the deformed quantum harmonic oscillators for these two types of deformation are defined as [4-6, 20, 21]:

$$
\hat{\mathrm{H}}_{q}=\left(\frac{\hbar \omega}{2}\right)\left(\hat{a}_{q} \hat{a}_{q}^{\dagger}+\hat{a}_{q}^{\dagger} \hat{a}_{q}\right)
$$

and,

$$
\begin{aligned}
\hat{\mathrm{H}}_{f}= & \left(\frac{\hbar \omega}{2}\right) \\
& \cdot\left(\hat{N} f^{2}(\hat{N})+(\hat{N}+1) f^{2}(\hat{N}+1)\right)
\end{aligned}
$$

respectively.

Eqn. (5) represents the Hamiltonian operator of the q-deformed quantum harmonic oscillator in the q-deformed Fock space while eqn. (6) represents the Hamiltonian operator of the f-deformed quantum harmonic oscillator in the undeformed Fock space.

The q-deformed number operator, $\hat{N}_{q}$, in terms of q-deformed boson operators is defined as $[1,15,19]$ :

$$
\hat{N}_{q}=[\hat{N}]_{q}=\hat{a}_{q}^{\dagger} \hat{a}_{q}
$$




$$
[\hat{N}+1]_{q}=\hat{a}_{q} \hat{a}_{q}^{\dagger}
$$

Substituting eqns. (7) and (8) in eqn. (6), one gets [15]:

Zaslavskii's Method for Deriving the Quantum Liouville Equation for the q-Deformed Oscillator in the $\alpha$-Representation

According to Zaslavskii [13], the equation of motion of an averaged physical quantity $\xi\left(\alpha, \alpha^{*} ; t\right)$ is defined as [13]:

$$
\frac{\text { \ } \xi\left(\alpha, \alpha^{*} ; t\right)}{\text { \ } t}=-i \hat{\mathrm{K}} \xi\left(\alpha, \alpha^{*} ; t\right)
$$

where,

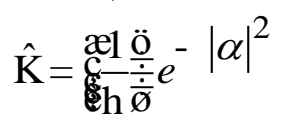

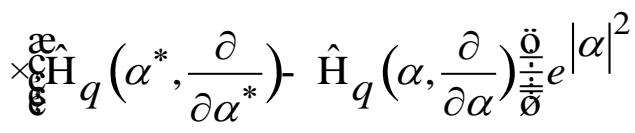

The Hamiltonian of the q-deformed quantum harmonic oscillator is given by

$$
\hat{\mathrm{H}}_{q}=\left(\frac{\hbar \omega}{2}\right)\left([\hat{N}]_{q}+[\hat{N}+1]_{q}\right)
$$

eqn. (9). And according to Sudarshan et al. [22], one have the following correspondence:

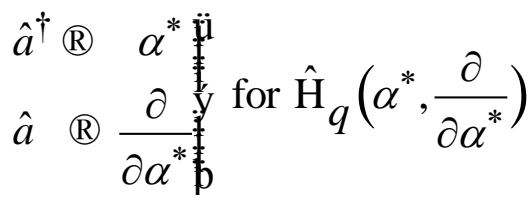

and,

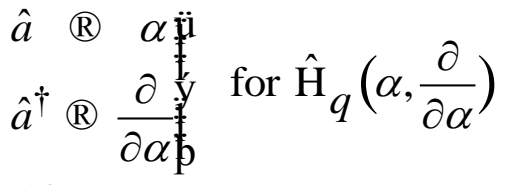

Substituting eqns. (12) and (13) into the expressions for $[\hat{N}]_{q}$ and $[\hat{N}+1]_{q}$ appearing in $\hat{\mathrm{H}}_{q}\left(\alpha^{*}, \frac{\partial}{\partial \alpha^{*}}\right)$ (see eqn. (9)), leads

to:

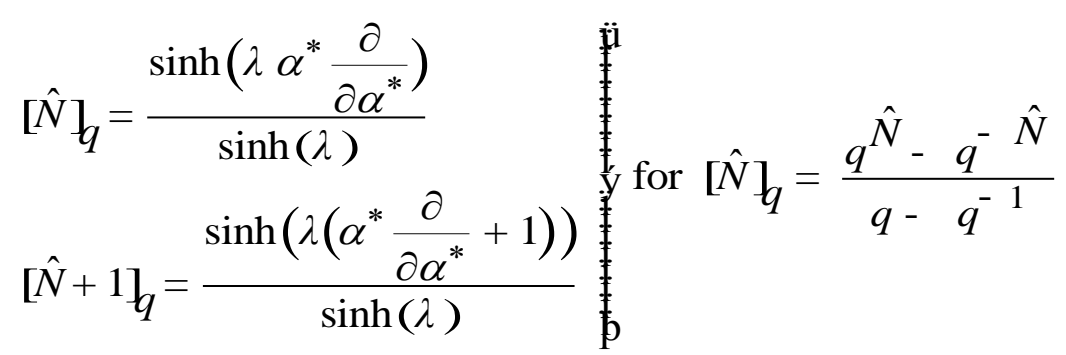

and,

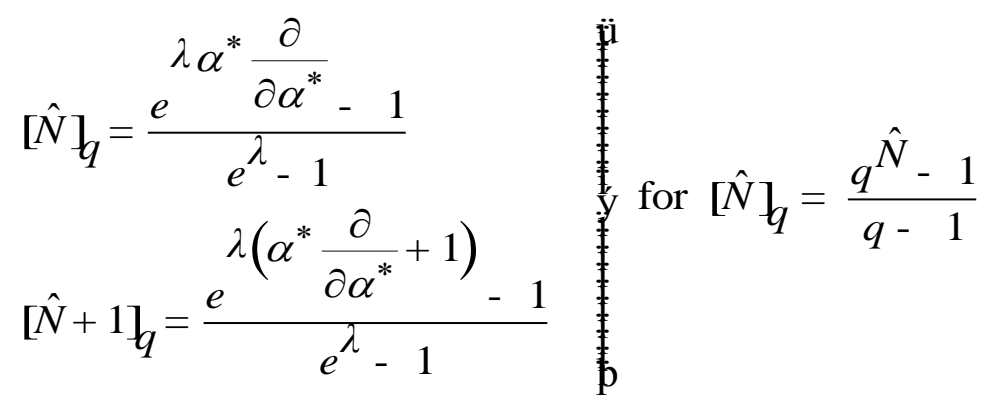

where, $[\hat{N}]_{q}$ and $[\hat{N}+1]_{q}$ given by eqns. (7) and (8). The same method can be used for $[\hat{N}]_{q}$ and $[\hat{N}+1]_{q}$ appearing in $\hat{\mathrm{H}}_{q}\left(\alpha, \frac{\partial}{\partial \alpha}\right)$. Now, substitution of $[\hat{N}]_{q}$ and $[\hat{N}+1]_{q}$ from eqns. (14) and (15) into eqn. (9), gives: 


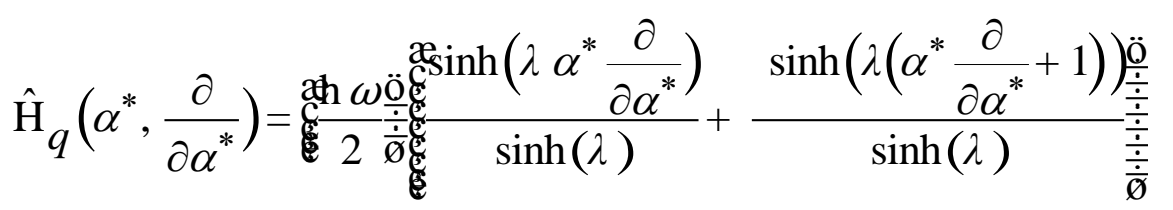

hence,

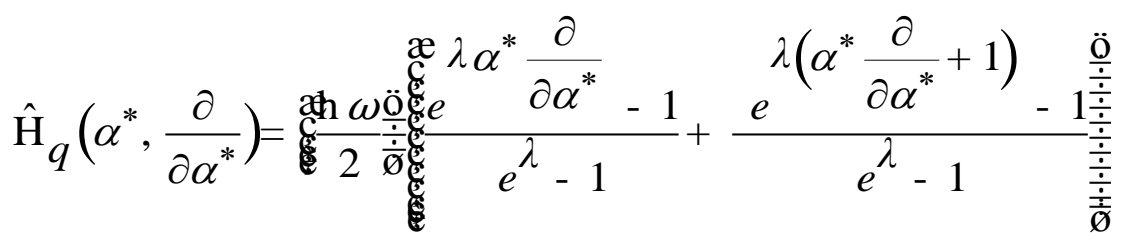

and,

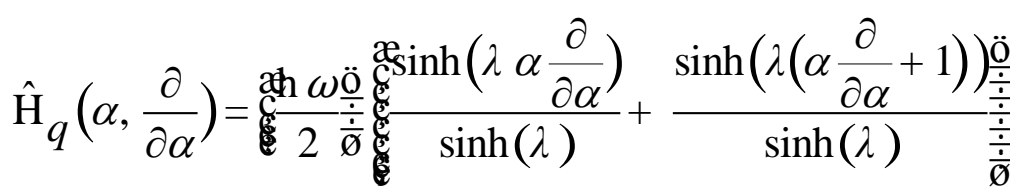

hence,

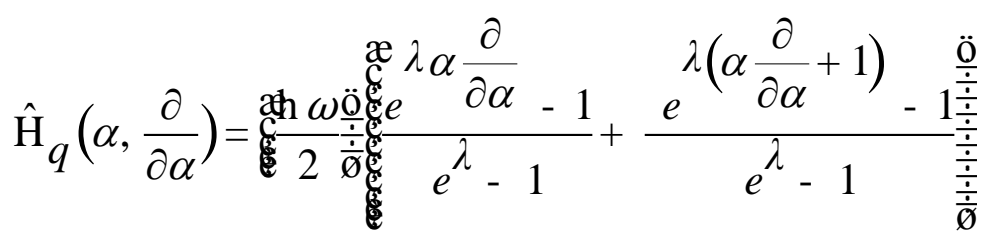

respectively.

Using eqns. (16) and (17) in eqn. (11), leads to:

$$
\begin{aligned}
& \hat{\mathrm{K}}=\omega(2 \sinh (\lambda))^{-1} e^{-|\alpha|^{2}} \\
& \underset{1}{x_{1}^{+}} \sinh \left(\lambda \alpha^{*} \frac{\partial}{\partial \alpha^{*}}\right)+\sinh \left(\lambda\left(\alpha^{*} \frac{\partial}{\partial \alpha^{*}}+1\right)\right) \\
& -\sinh \left(\lambda \alpha \frac{\partial}{\partial \alpha}\right)-\sinh \left(\lambda\left(\alpha \frac{\partial}{\partial \alpha}+1\right)\right) \frac{\hat{p}_{p}^{i}}{p}
\end{aligned}
$$$$
\times e^{|\alpha|^{2}}
$$

and,

$$
\begin{aligned}
& \hat{\mathrm{K}}=\omega\left(2\left(e^{\lambda}-1\right)\right)^{-1} e^{-|\alpha|^{2}}
\end{aligned}
$$

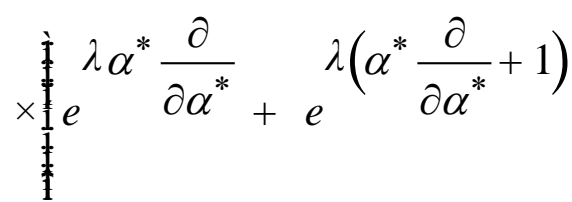

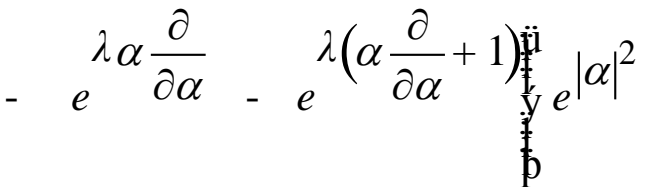

respectively.

Then, using the definition of the sine hyperbolic function and re-arranging terms, eqn. (18a) can be cast in the form: 


$$
\begin{aligned}
& \hat{\mathrm{K}}=\omega(4 \sinh (\lambda))^{-1} e^{-|\alpha|^{2}}
\end{aligned}
$$

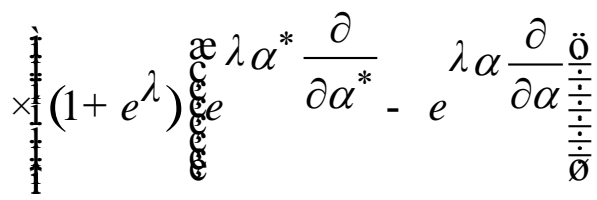

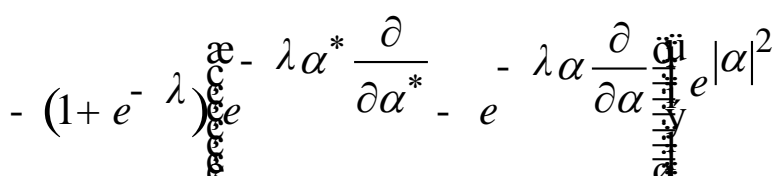

Similarly, eqn. (18b) becomes:

$$
\begin{aligned}
& \frac{\text { \ } \xi_{q}\left(\alpha, \alpha^{*} ; t\right)}{\text { \t}}=\omega(4 \sinh (\lambda))^{-1} e^{-|\alpha|^{2}}
\end{aligned}
$$

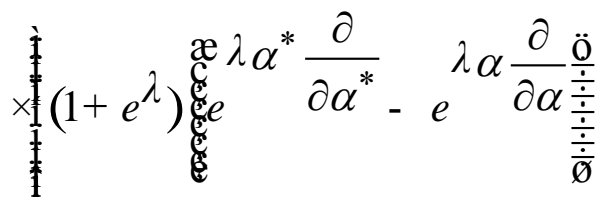

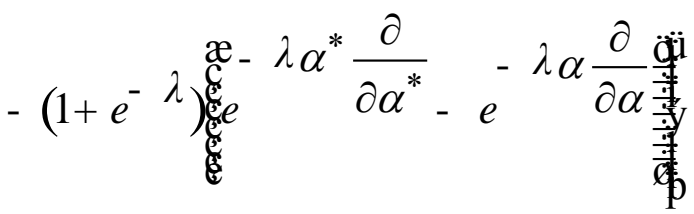

$$
\begin{aligned}
& \times e^{|\alpha|^{2}} \xi_{q}\left(\alpha, \alpha^{*} ; t\right)
\end{aligned}
$$

and,

$$
\begin{aligned}
& \frac{\text { \ } \xi_{q}\left(\alpha, \alpha^{*} ; t\right)}{\text { \t} t}=\omega\left(2\left(e^{\lambda}-1\right)\right)^{-1} e^{-|\alpha|^{2}}
\end{aligned}
$$

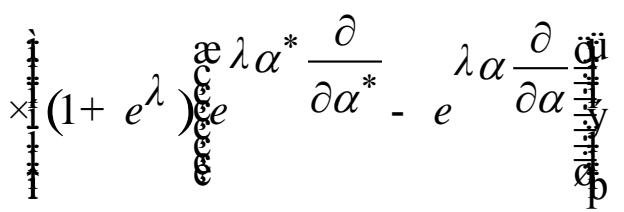

$$
\begin{aligned}
& \times e^{|\alpha|^{2}} \xi_{q}\left(\alpha, \alpha^{*} ; t\right)
\end{aligned}
$$

respectively, where the function $\xi_{q}\left(\alpha, \alpha^{*} ; t\right)$ represents the q-analog of the averaged physical quantity

$$
\begin{aligned}
& \hat{\mathrm{K}}=\omega\left(2\left(e^{\lambda}-1\right)\right)^{-1} e^{-|\alpha|^{2}}
\end{aligned}
$$

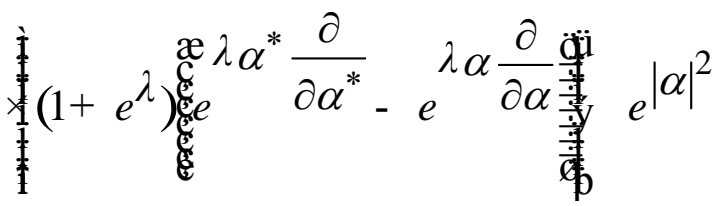

Eqns. (19a) and (19b) can be substituted in eqn. (10), and after replacing $\xi\left(\alpha, \alpha^{*} ; t\right)$ by $\xi_{q}\left(\alpha, \alpha^{*} ; t\right)$, one obtains:

$\xi\left(\alpha, \alpha^{*} ; t\right)$ appearing in ref. [13]. Also, it is noted that the function $\xi_{q}\left(\alpha, \alpha^{*} ; t\right)$ reduces to $\xi\left(\alpha, \alpha^{*} ; t\right)$ in the limit $q \rightarrow 1$.

\section{Action of the Dilatation (Shift)}

Operators $e^{ \pm \lambda \alpha^{*} \frac{\partial}{\partial \alpha^{*}}}$ and $e^{ \pm \lambda \alpha \frac{\partial}{\partial \alpha}}$ on the Function $\xi_{q}\left(\alpha, \alpha^{*} ; t\right)$

Since the action of the dilatation (shift) operator $e^{b x \frac{\|}{\Phi x}}$ on a function $F(x)$ is given by $[23,24]$ :

$e^{\beta x \frac{\partial}{\partial x}} F(x)=F\left(x e^{\beta}\right)$

for any arbitrary constant $b= \pm \lambda$, and replacing $F(x)$ by $F\left(\alpha, \alpha^{*}\right)$, eqn. (21) gives:

$$
e^{ \pm \lambda \alpha^{*} \frac{\partial}{\partial \alpha^{*}}} F\left(\alpha, \alpha^{*}\right)=F\left(\alpha, e^{ \pm \lambda} \alpha^{*}\right)
$$

and,

$$
e^{ \pm \lambda \alpha \frac{\partial}{\partial \alpha}} F\left(\alpha, \alpha^{*}\right)=F\left(e^{ \pm \lambda} \alpha, \alpha^{*}\right)
$$


Eqns. (22) and (23) can be generalized to the case where the shift operator acts on the product of two functions $F\left(\alpha, \alpha^{*}\right) G\left(\alpha, \alpha^{*}\right)$ (see Appendix).

Using this result, leads to:

$$
\begin{aligned}
& e^{ \pm \lambda \alpha^{*} \frac{\partial}{\partial \alpha^{*}}} F\left(\alpha, \alpha^{*}\right) G\left(\alpha, \alpha^{*}\right)= \\
& F\left(\alpha, e^{ \pm \lambda} \alpha^{*}\right) e^{ \pm \lambda \alpha^{*} \frac{\partial}{\partial \alpha^{*}}} G\left(\alpha, \alpha^{*}\right)
\end{aligned}
$$

and,

$\frac{\prod \xi_{q}\left(\alpha, \alpha^{*} ; t\right)}{\operatorname{q} t}=(-i \omega)(4 \sinh (\lambda))^{-1} e^{-|\alpha|^{2}}$

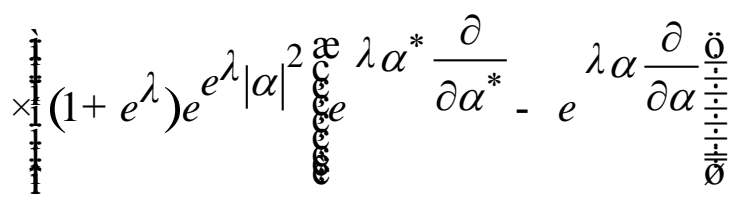

$$
\begin{aligned}
& -\left(1+e^{-\lambda}\right) e^{e^{-\lambda}}|\alpha|^{2}
\end{aligned}
$$

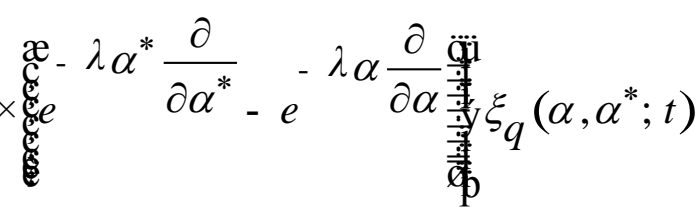

and,

$$
\begin{aligned}
& \frac{\prod \xi_{q}\left(\alpha, \alpha^{*} ; t\right)}{\mathbb{T} t}=(-i \omega)\left(2\left(e^{\lambda}-1\right)\right)^{-1} e^{-|\alpha|^{2}}
\end{aligned}
$$

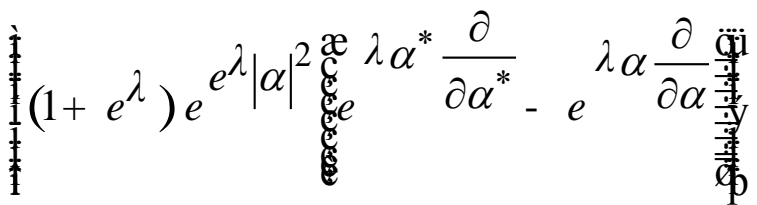

$$
\begin{aligned}
& \times \xi_{q}\left(\alpha, \alpha^{*} ; t\right)
\end{aligned}
$$

respectively.

Eqns. (26) represent the quantum Liouville equations for the q-deformed 1-D quantum

$$
\begin{aligned}
& e^{ \pm \lambda \alpha \frac{\partial}{\partial \alpha}} F\left(\alpha, \alpha^{*}\right) G\left(\alpha, \alpha^{*}\right)= \\
& \quad F\left(e^{ \pm \lambda} \alpha, \alpha^{*}\right) e^{ \pm \lambda \alpha \frac{\partial}{\partial \alpha}} G\left(\alpha, \alpha^{*}\right)
\end{aligned}
$$

respectively.

Therefore, eqns. (20) can be simplified by using eqns. (24) and (25) with $F\left(\alpha, \alpha^{*}\right)=e^{|\alpha|^{2}}, G\left(\alpha, \alpha^{*}\right)=\xi_{q}\left(\alpha, \alpha^{*} ; t\right)$ and re-arranging to get:

harmonic oscillator in the $\alpha$-representation.

$$
\begin{aligned}
& \cdot\left[\left(\alpha^{*} \frac{\partial}{\partial \alpha^{*}}-\alpha \frac{\partial}{\partial \alpha}\right)\right. \\
& \left.+\frac{\lambda}{2 !}\left(\left(\alpha^{*} \frac{\partial}{\partial \alpha^{*}}\right)^{2}-\left(\alpha \frac{\partial}{\partial \alpha}\right)^{2}\right)+\cdots\right] \\
& +\left(2+S_{3}(\lambda)\right) e^{\left(-\lambda|\alpha|^{2}+\frac{\lambda^{2}}{2 !}|\alpha|^{2}-\cdots\right)} \\
& \cdot\left[\left(\alpha^{*} \frac{\partial}{\partial \alpha^{*}}-\alpha \frac{\partial}{\partial \alpha}\right)\right.
\end{aligned}
$$

$$
\left.\left.-\frac{\lambda}{2 !}\left(\left(\alpha^{*} \frac{\partial}{\partial \alpha^{*}}\right)^{2}-\left(\alpha \frac{\partial}{\partial \alpha}\right)^{2}\right)+\cdots\right]\right\}
$$

where, 


$$
\left.\begin{array}{l}
S_{1}(\lambda)=\frac{\lambda^{2}}{3 !}+\frac{\lambda^{4}}{5 !}+\frac{\lambda^{6}}{7 !}+\cdots \\
S_{2}(\lambda)=\lambda+\frac{\lambda^{2}}{2 !}+\frac{\lambda^{3}}{3 !}+\cdots \\
S_{3}(\lambda)=-\lambda+\frac{\lambda^{2}}{2 !}-\frac{\lambda^{3}}{3 !}+\cdots
\end{array}\right\}
$$

\section{Classical Limit of the Liouville}

Equation for $\xi_{q}\left(\alpha, \alpha^{*} ; t\right)$ in the $\alpha$ -

\section{Representation}

Expanding all functions appearing in eqn. (26a) as power series in $\lambda$, and simplifying the result, one obtains:

$$
\begin{aligned}
& \frac{\partial \xi_{q}\left(\alpha, \alpha^{*} ; t\right)}{\partial t}=-\left(\frac{i \omega}{4}\right)\left(\frac{1}{\left(1+S_{1}(\lambda)\right)}\right) \\
& \cdot\left\{\left(2+S_{2}(\lambda)\right) e^{\left(\lambda|\alpha|^{2}+\frac{\lambda^{2}}{2 !}|\alpha|^{2}+\cdots\right)}\right. \\
& \frac{\partial \mathscr{P}_{C L}^{q}\left(\alpha, \alpha^{*} ; t\right)}{\partial t}=-\left(\frac{i \omega}{4}\right) \\
& \cdot\left\{\left\{2 e^{\lambda|\alpha|^{2}}\left(\alpha^{*} \frac{\partial}{\partial \alpha^{*}}-\alpha \frac{\partial}{\partial \alpha}\right)\right.\right. \\
& \left.+2 e^{-\lambda|\alpha|^{2}}\left(\alpha^{*} \frac{\partial}{\partial \alpha^{*}}-\alpha \frac{\partial}{\partial \alpha}\right)\right\} \\
& \cdot \mathscr{P}_{C L}^{q}\left(\alpha, \alpha^{*} ; t\right)
\end{aligned}
$$

where $\mathscr{P}_{C L}^{q}\left(\alpha, \alpha^{*} ; t\right) \quad$ represents the classical probability distribution function. It should be noticed that the fact that in this limit $S_{1}(\lambda)=S_{2}(\lambda)=S_{3}(\lambda)=0$ has been used. Re-arranging the terms in eqn. (29), this equation becomes:
Applying the conditions for classical limiting namely; $h \rightarrow 0,|\alpha|^{2} \rightarrow \infty$ such that $\lambda|\alpha|^{2} \rightarrow$ finite, to eqn. (28), where $\lambda=($ const. $) \cdot \mathrm{h}$ (i.e., $\lambda \rightarrow 0$ as fast as $\mathrm{h} \rightarrow 0) \quad$ and letting $\xi_{q}\left(\alpha, \alpha^{*} ; t\right) \rightarrow \mathcal{P}_{C L}^{q}\left(\alpha, \alpha^{*} ; t\right) \quad$ in this limit, then eqn. (27) reduces to:

$$
\begin{aligned}
& \frac{\partial \mathscr{P}_{C L}^{q}\left(\alpha, \alpha^{*} ; t\right)}{\partial t}= \\
& -i \omega_{q}^{(1)}\left(\alpha^{*} \frac{\partial}{\partial \alpha^{*}}-\alpha \frac{\partial}{\partial \alpha}\right) \mathscr{P}_{C L}^{q}\left(\alpha, \alpha^{*} ; t\right)
\end{aligned}
$$

where,

$$
\omega_{q}^{(\mu)}=\omega \cosh \left(\lambda|\alpha|^{2}\right)
$$

Eqn. (30) represents a classical Liouville equation for a classical harmonic oscillator having frequency $\omega_{q}^{(1)}$. By expanding the frequency of this oscillator $\omega_{q}^{(1)}$ up to $\lambda^{2}$, eqn. (30) becomes: 


$$
\begin{gathered}
\frac{\partial \mathcal{P}_{C L}^{q}\left(\alpha, \alpha^{*} ; t\right)}{\partial t}=-i \omega\left\{\left(\alpha^{*} \frac{\partial}{\partial \alpha^{*}}-\alpha \frac{\partial}{\partial \alpha}\right)\right. \\
\left.\left.+\frac{\lambda^{2}}{2 !}|\alpha|^{4}\left(\alpha^{*} \frac{\partial}{\partial \alpha^{*}}-\alpha \frac{\partial}{\partial \alpha}\right)\right)\right\} P_{C L}^{q}\left(\alpha, \alpha^{*} ; t\right)
\end{gathered}
$$

Eqn. (32) can be interpreted as a classical Liouville equation for a classical harmonic

oscillator with frequency:

$$
\omega_{q}^{(2)}=\omega\left(1+\frac{\lambda^{2}}{2 !}|\alpha|^{4}\right)
$$

Similarly, eqn. (26b) gives:

$$
\begin{aligned}
& \frac{\partial \mathscr{P}_{C L}^{q}\left(\alpha, \alpha^{*} ; t\right)}{\partial t}=-i \omega_{q}^{(3)} \\
& \cdot\left(\alpha^{*} \frac{\partial}{\partial \alpha^{*}}-\alpha \frac{\partial}{\partial \alpha}\right) \mathscr{P}_{C L}^{q}\left(\alpha, \alpha^{*} ; t\right) \\
& \omega_{q}^{(\mu)}=\omega e^{\lambda|\alpha|^{2}}
\end{aligned}
$$

Also, by expanding the frequency, $\omega_{q}^{(3)}$, of this oscillator up to $\lambda$, and applying the

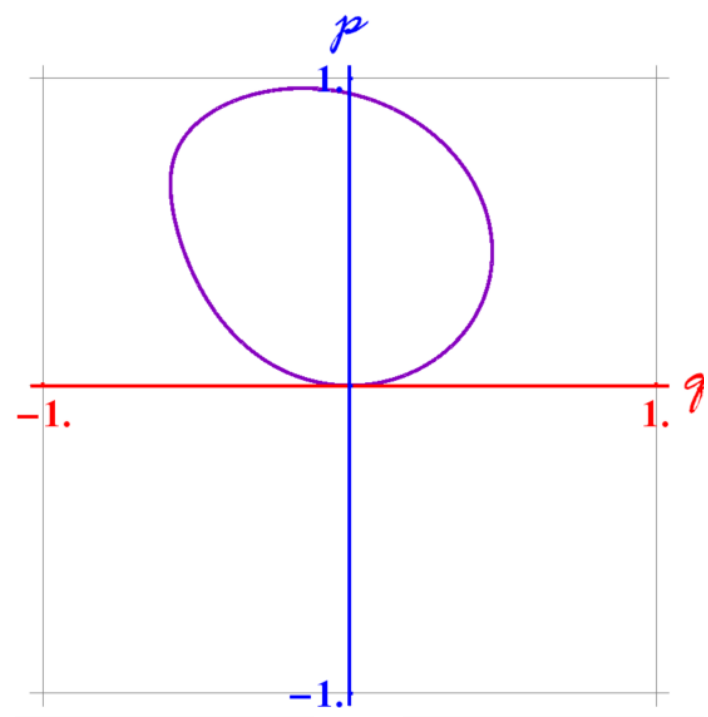

a.

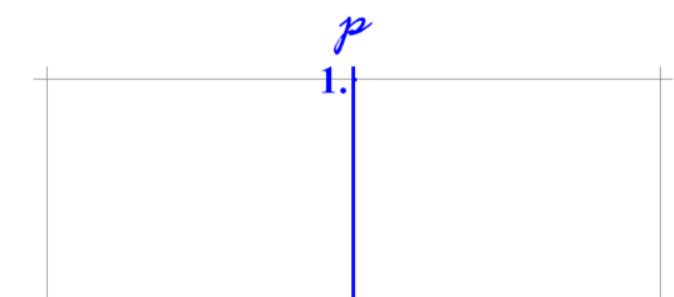

same previous mentioned limiting conditions, eqn. (34) becomes:

$$
\begin{aligned}
& \frac{\partial P_{C L}^{q}\left(\alpha, \alpha^{*} ; t\right)}{\partial t}=-i \omega_{q}^{(4)} \\
& \cdot\left(\alpha^{*} \frac{\partial}{\partial \alpha^{*}}-\alpha \frac{\partial}{\partial \alpha}\right) P_{C L}^{q}\left(\alpha, \alpha^{*} ; t\right)
\end{aligned}
$$

where,

$$
\omega_{q}^{(\mu)}=\omega\left(1+\lambda|\alpha|^{2}\right)
$$

By using the same technique that was introduced in Ref. [25], eqns. (30), (34) and (36) can be solved by the method of characteristics, and the time-evolution of the classical probability distribution function can be investigated in the nonrotating frame in phase space via a computer visualization method [25]. The results can then be shown in a 2-dimensional time-evolution contours of the probability distribution functions $\mathbb{P}_{C L}^{q}\left(\alpha, \alpha^{*} ; t\right)$ in phase space. These probability distributions functions exhibit whorl shapes and it is obvious that these whorl shapes become finer as $t \rightarrow \infty$ as in Figs. (1) - (3).

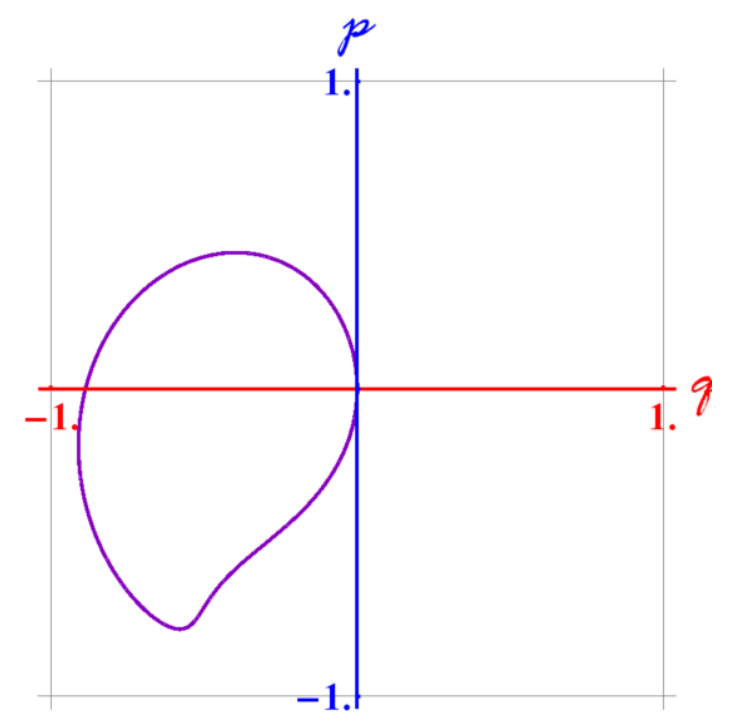

b. 


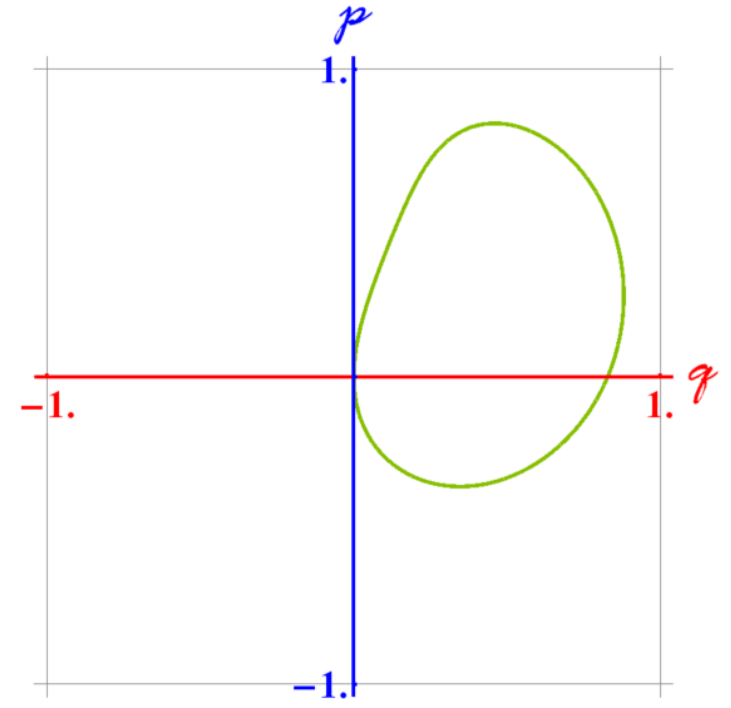

a.

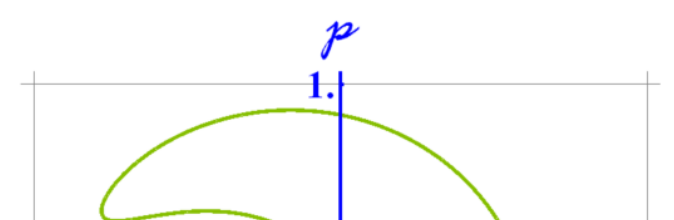

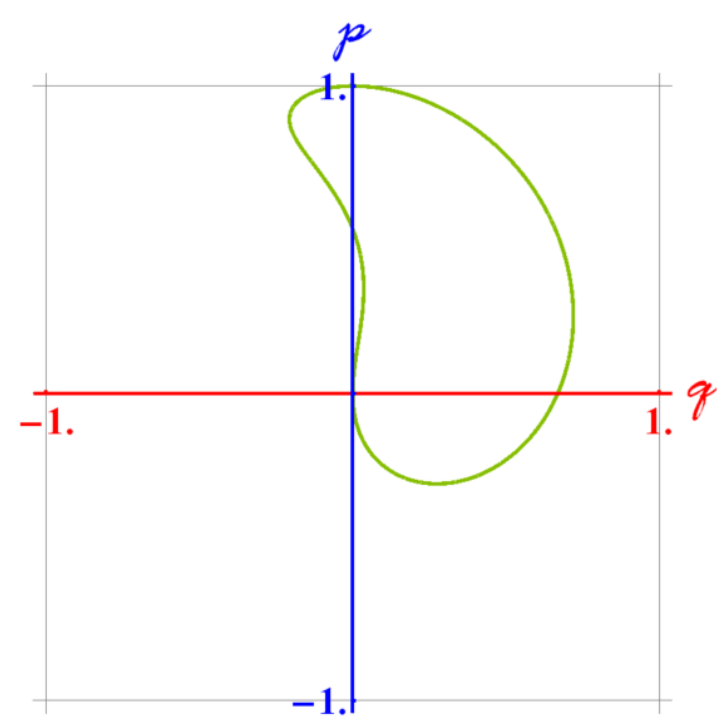

b.

0

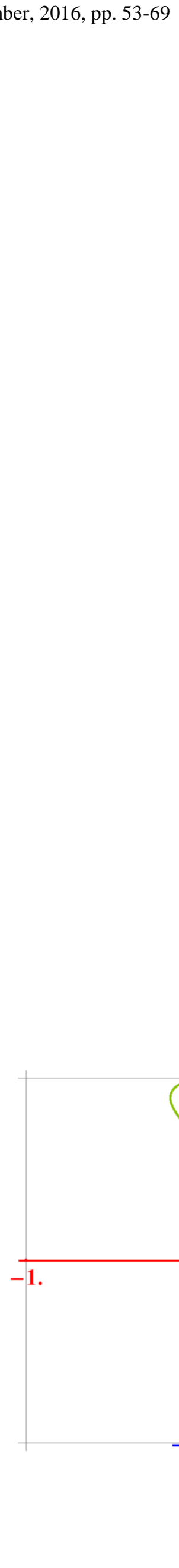

$p$ 


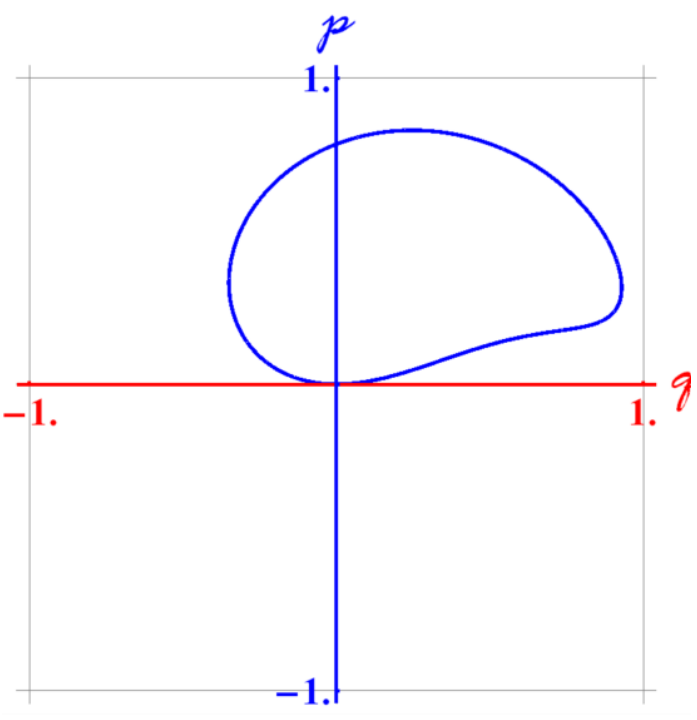

a.

11

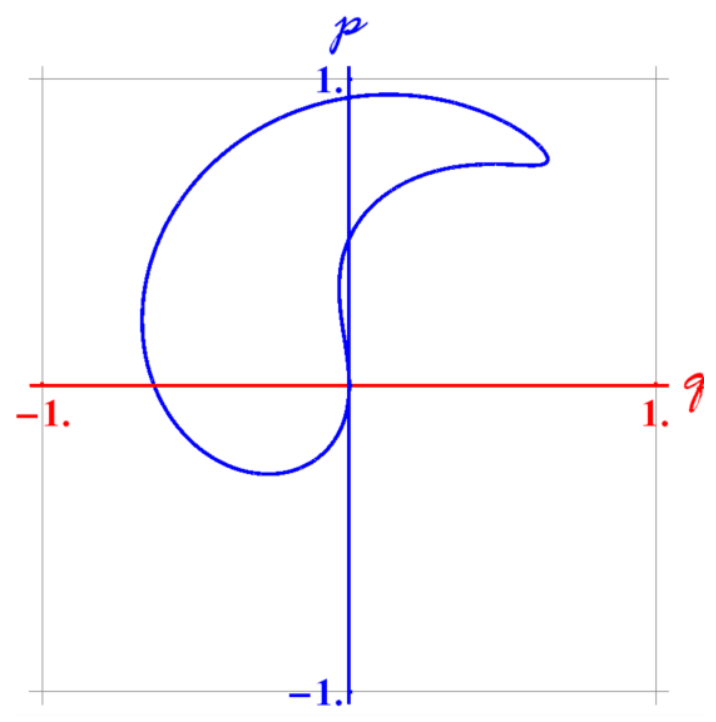

b.

$p$

1 
Also, in Figs (4) - (6), the results of the 3D time-evolution of the classical probability distribution functions $\mathbb{P}_{C L}^{q}\left(\alpha, \alpha^{*} ; t\right)$ are presented in phase space. From these figures, it can be seen that these probability distributions appear as q-deformed Gaussians. It is also clear from all these figures that the peaks of these q-deformed Gaussians do not change with time. These peaks follow the classical trajectories for the probability distribution functions shown in Figs. (1)-(3). Another observation is that the Gaussian shapes of these distributions become more convoluted around themselves as $t \rightarrow \infty$.
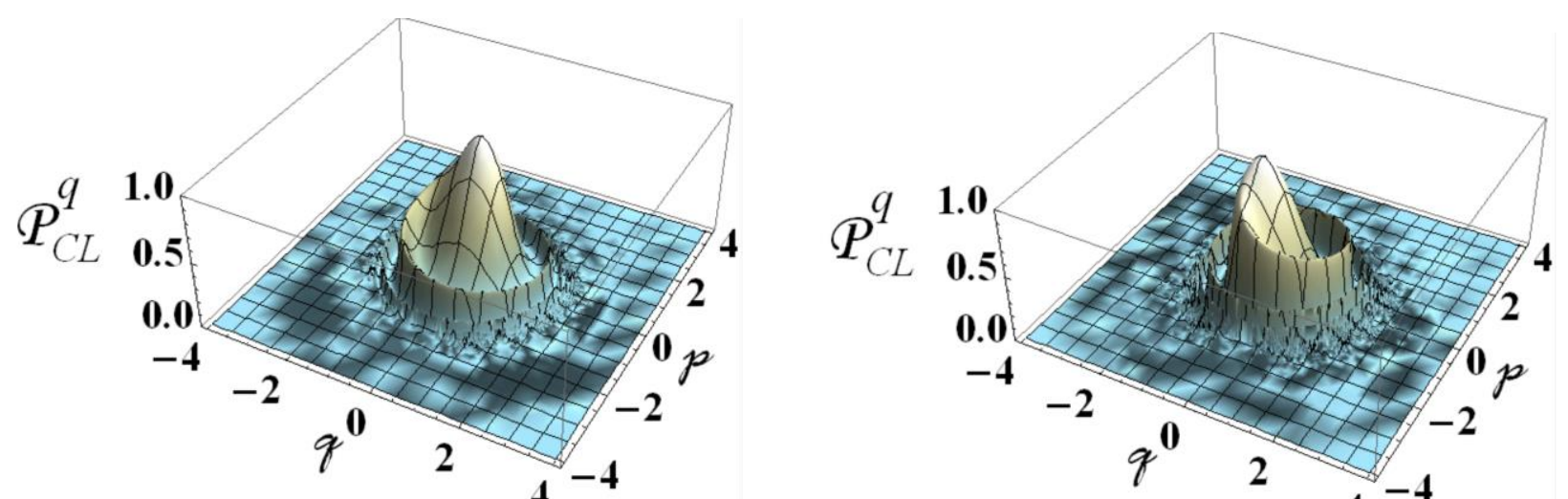


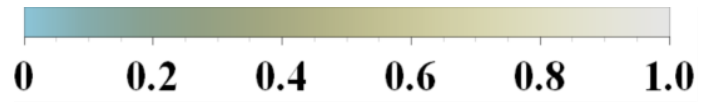

a.

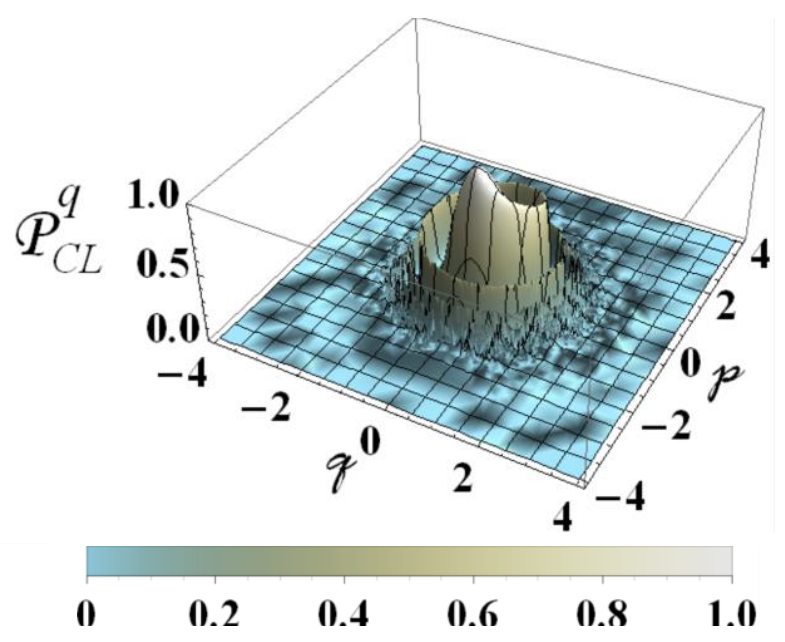

c.

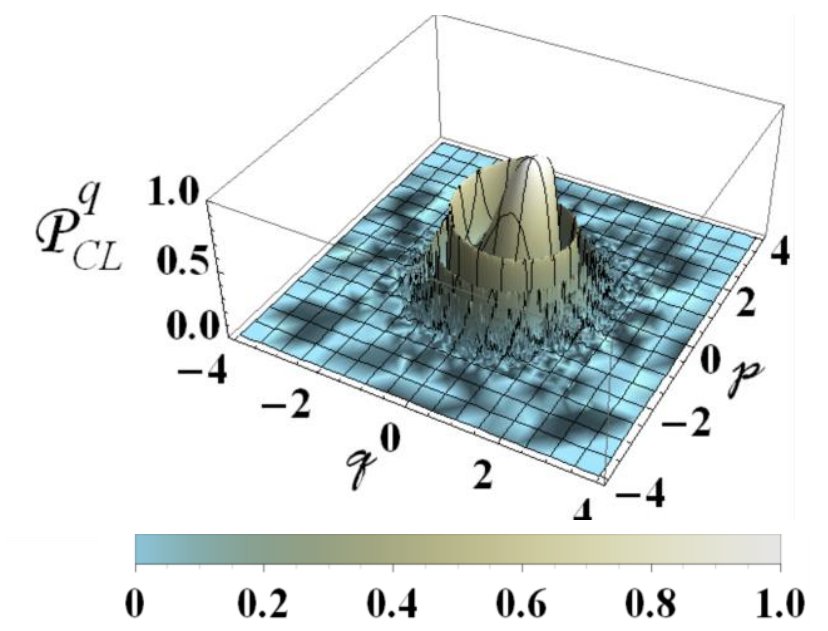

d.

Fig.(4): The 3-D time-evolution of the classical probability distribution function $P_{C L}^{q}\left(\alpha, \alpha^{*} ; t\right)$ for the q-deformed harmonic oscillator with frequency $\omega_{q}^{(1)}$ given by eqn. (31) and $q=0.5$ in phase space, for different values of time ( $\tau$ ): (a) $\tau=\pi / 2$, (b) $\tau=\pi$, (c) $\tau=3 \pi / 2$, and (d) $\tau=2 \pi$.

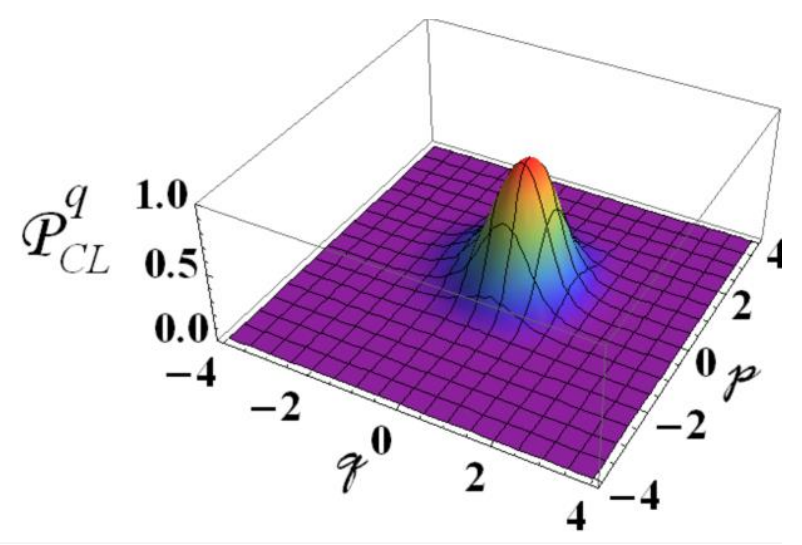

$\begin{array}{llllll}0 & 0.2 & 0.4 & 0.6 & 0.8 & 1.0\end{array}$

a.

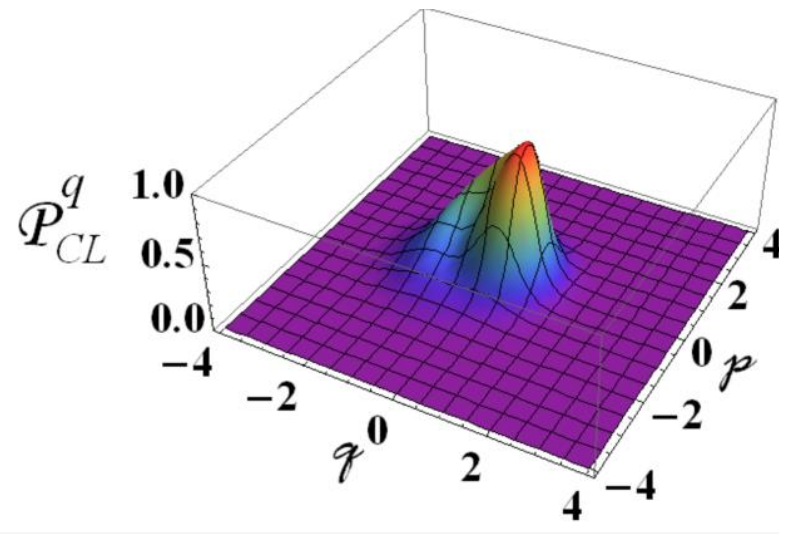

b. 


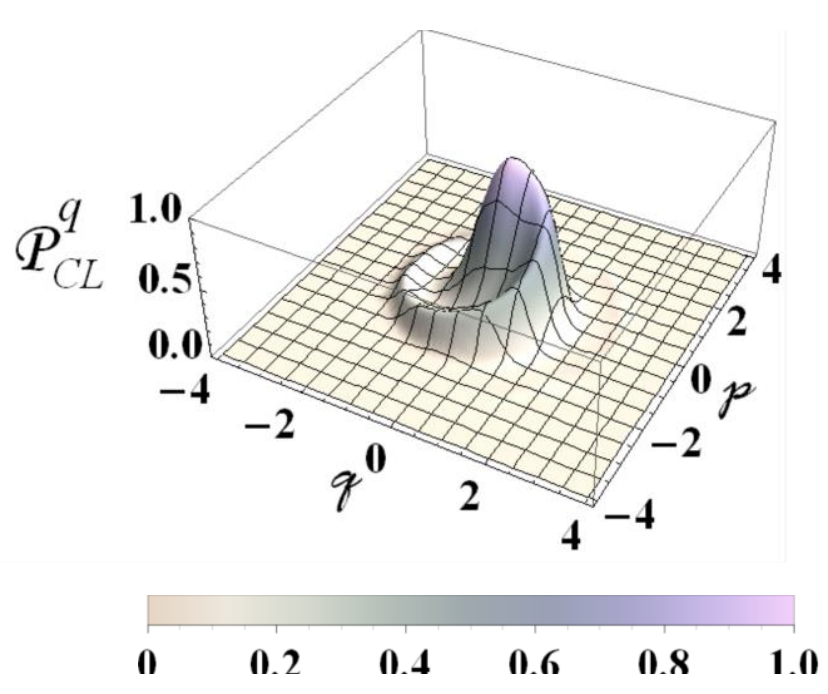

a.

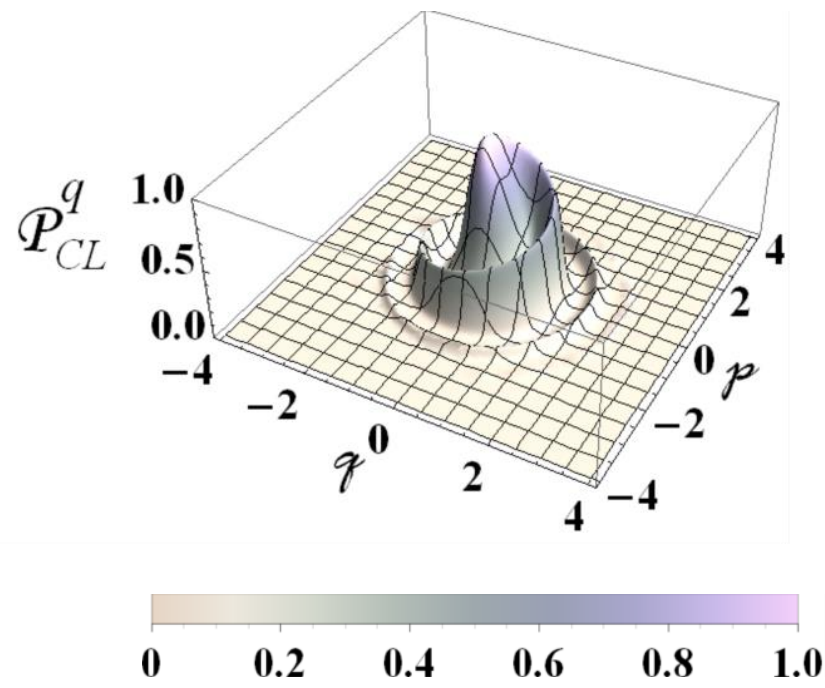

b. 


\section{Conclusions}

A number of conclusions can be drawn from the present investigation as follows:

1. The classical limit of the 1-D qdeformed quantum harmonic oscillator is statistical in nature. This is clear from eqns. (32) and (36) where the classical Liouville equations are obtained for the 1D q-deformed classical harmonic oscillator in the $\quad \alpha$-representation. This is in conformity with the work of Ghosh et al. [26], where the classical Liouville equation was obtained for the 1-D classical simple harmonic oscillator by applying the classical limiting conditions $\hbar \rightarrow 0$, $|\alpha|^{2} \rightarrow \infty$, such that $\hbar|\alpha|^{2} \rightarrow$ finite .

2. The q-deformed 1-D quantum harmonic oscillator can be interpreted as a nonlinear quantum oscillator where the nonlinearity parameter $\lambda$ depends on the $h$ such that $\lambda=($ const. $) \cdot \mathrm{h}$. This dependence is required for the classical limit to exist. Based on the more detailed approach to the classical limit adopted in this work, this interpretation seems to be more accurate than that introduced by Man'ko [4] where this oscillator was interpreted as a nonlinear quantum oscillator with a special 
type of nonlinearity with an energy dependent frequency.

3. The q-deformation of the 1-D quantum harmonic oscillator induces a non-commutative geometry. This can be understood in the light of Vitiello's work [27], where the q-deformation of the coherent states was studied to find that the fractal self-similarity obtained by defining a fractal operator $q^{\alpha \frac{d}{d \alpha}}$ leads to a non-commutative geometry. The expression of this fractal operator is similar to those appearing in the present work as a dilatation (shift) operators $e^{ \pm \lambda \alpha \frac{\partial}{\partial \alpha}}$ and $e^{ \pm \lambda \alpha^{*} \frac{\partial}{\partial \alpha^{*}}}$. These dilatation (shift) operators are inherent in the q-deformation and arise naturally in the quantum Liouville equations given in eqns. (26), for the q-deformed 1-D quantum harmonic oscillator in the $\alpha$ representation.

4. The behavior of the classical limit of the quantum Liouville equation for the q-deformed 1-D quantum harmonic oscillator in phase space shows whorl shapes evolving with time as in Figs. (1)-(3). These figures are similar to those introduced by Milburn [28] for the 1-D classical anharmonic oscillator. This similarity results from the fact that the anharmonicity itself represents a kind of

$$
\begin{aligned}
& F(x)=\sum_{\mathrm{m}=0}^{\infty} a_{\mathrm{m}} x^{\mathrm{m}} \\
& G(x)=\sum_{\mathrm{m}=0}^{\infty} b_{\mathrm{m}} x^{\mathrm{m}}
\end{aligned}
$$

Using these expressions, the product $F(x) G(x)$ can be written as:

$$
\begin{aligned}
F(x) G(x) & =\left(a_{o}+a_{1} x+a_{2} x^{2}+a_{3} x^{3}+\cdots\right) \\
& \cdot\left(b_{o}+b_{1} x+b_{2} x^{2}+b_{3} x^{3}+\cdots\right)
\end{aligned}
$$

deformation with a frequency which is a function of. The significance of this observation lies in the assumption that the whorl shapes in phase space can be considered as a generalized phenomenon whenever the q-deformation is used for any quantum system with arbitrary potential.

\section{Acknowledgment:}

The authors would like to thank Dr. Salam S. Tawfiq, from the Dept. of Physics, University of Toronto, Canada for supplying them with necessary literature.

\section{Appendix}

\section{The action of the dilatation (shift)} operators

$$
\begin{gathered}
e^{ \pm \lambda \alpha \frac{\partial}{\partial \alpha}} F\left(\alpha, \alpha^{*}\right) G\left(\alpha, \alpha^{*}\right) \text { and } \\
e^{ \pm \lambda \alpha^{*} \frac{\partial}{\partial \alpha^{*}}} F\left(\alpha, \alpha^{*}\right) G\left(\alpha, \alpha^{*}\right)
\end{gathered}
$$

Assume two functions $F(x)$ and $G(x)$ that have power series expressions of the form:

Multiplying both sides of eqn. (A.3) from left by the dilatation (shift) operator $e^{\beta x \frac{\partial}{\partial x}}$ where $\beta$ and $x$ are given in eqns. (21), 


$$
\begin{aligned}
& +a_{1}\left(e^{\beta} x\right)\left(b_{o}+b_{1}\left(e^{\beta} x\right)+b_{2}\left(e^{\beta} x\right)^{2}\right. \\
& \left.+b_{3}\left(e^{\beta} x\right)^{3}+\cdots\right) \\
& +a_{2}\left(e^{\beta} x\right)^{2}\left(b_{o}+b_{1}\left(e^{\beta} x\right)+b_{2}\left(e^{\beta} x\right)^{2}\right. \\
& \left.+b_{3}\left(e^{\beta} x\right)^{3}+\cdots\right) \\
& +a_{3}\left(e^{\beta} x\right)^{3}\left(b_{o}+b_{1}\left(e^{\beta} x\right)+b_{2}\left(e^{\beta} x\right)^{2}\right. \\
& \left.+b_{3}\left(e^{\beta} x\right)^{3}+\cdots\right)
\end{aligned}
$$

the result is:

$$
\begin{aligned}
& e^{\beta x \frac{\partial}{\partial x}} F(x) G(x)=e^{\beta x \frac{\partial}{\partial x}} \\
& \cdot\left\{\left(a_{o} b_{o}+a_{o} b_{1} x+a_{o} b_{2} x^{2}\right.\right. \\
& \left.+a_{o} b_{3} x^{3}+\cdots\right) \\
& +\left(a_{1} b_{o} x+a_{1} b_{1} x^{2}+a_{1} b_{2} x^{3}+\right. \\
& \left.+a_{1} b_{3} x^{4}+\cdots\right) \\
& +\left(a_{2} b_{o} x^{2}+a_{2} b_{1} x^{3}+a_{2} b_{2} x^{4}\right. \\
& \left.+a_{2} b_{3} x^{5}+\cdots\right) \\
& \left.+\left(a_{3} b_{o} x^{3}+a_{3} b_{1} x^{4}+a_{3} b_{2} x^{5}+a_{3} b_{3} x^{6}+\cdots\right)\right\}
\end{aligned}
$$

Applying eqn. (21) to eqn. (A.4) and simplifying, the result becomes:

$$
\begin{aligned}
& e^{\beta x \frac{\partial}{\partial x}} F(x) G(x)= \\
& a_{o}\left(b_{o}+b_{1}\left(e^{\beta} x\right)+b_{2}\left(e^{\beta} x\right)^{2}\right. \\
& \left.+b_{3}\left(e^{\beta} x\right)^{3}+\cdots\right)
\end{aligned}
$$

Collecting similar terms, this gives:

$$
\begin{aligned}
& e^{\beta x \frac{\partial}{\partial x}} F(x) G(x)= \\
& \left(a_{o}+a_{1}\left(e^{\beta} x\right)+a_{2}\left(e^{\beta} x\right)^{2}+a_{3}\left(e^{\beta} x\right)^{3}+\cdots\right) \\
& \cdot\left(b_{o}+b_{1}\left(e^{\beta} x\right)+b_{2}\left(e^{\beta} x\right)^{2}+b_{3}\left(e^{\beta} x\right)^{3}+\cdots\right)
\end{aligned}
$$

But since,

$$
\begin{aligned}
& F\left(e^{\beta} x\right)=\sum_{\mathrm{m}=0}^{\infty} a_{\mathrm{m}}\left(e^{\beta} x\right)^{\mathrm{m}} \\
& G\left(e^{\beta} x\right)=\sum_{\mathrm{m}=0}^{\infty} b_{\mathrm{m}}\left(e^{\beta} x\right)^{\mathrm{m}}
\end{aligned}
$$

then, substituting eqns. (A.7) and (A.8) into eqn. (A.6), one obtains:

$$
e^{\beta x \frac{\partial}{\partial x}} F(x) G(x)=F\left(e^{\beta} x\right) G\left(e^{\beta} x\right)
$$

Using $\quad F(x) \rightarrow F\left(\alpha, \alpha^{*}\right)$ as given in eqn. (22) and similarly $G(x) \rightarrow G\left(\alpha, \alpha^{*}\right)$ in eqn. (A.9), substituting the definition of $\beta$ from eqn. (21) then applying eqns. (22), (23) for $F\left(\alpha, \alpha^{*}\right)$ and $G\left(\alpha, \alpha^{*}\right)$ respectively to eqn. (A.9), the results become: 


$$
\begin{aligned}
& e^{ \pm \lambda \alpha^{*} \frac{\partial}{\partial \alpha^{*}}} F\left(\alpha, \alpha^{*}\right) G\left(\alpha, \alpha^{*}\right)= \\
& F\left(\alpha, e^{ \pm \lambda} \alpha^{*}\right) e^{ \pm \lambda \alpha^{*} \frac{\partial}{\partial \alpha^{*}}} G\left(\alpha, \alpha^{*}\right)
\end{aligned}
$$

and,

$$
\begin{aligned}
& e^{ \pm \lambda \alpha \frac{\partial}{\partial \alpha}} F\left(\alpha, \alpha^{*}\right) G\left(\alpha, \alpha^{*}\right)= \\
& F\left(e^{ \pm \lambda} \alpha, \alpha^{*}\right) e^{ \pm \lambda \alpha \frac{\partial}{\partial \alpha}} G\left(\alpha, \alpha^{*}\right)
\end{aligned}
$$

\section{References}

[1] Buzek V.,

"Dynamics of a q-Analogue of the Quantum Harmonic Oscillator",

J. Mod. Opt., 38 (4), 801-812, 1991.

[2] Shabanov S. V.,

"The Poisson Bracket for qDeformed Systems",

J. Phys. A: Math. Gen., 25 (22),

L1245-L1250, 1992.

[3] Shabanov S. V.,

"Quantum and Classical

Mechanics of q-Deformed

Systems",

J. Phys. A: Math. Gen., 26 (11), 2583-2606, 1993.

[4] Man'ko V. I., Marmo G., Solimeno S. and Zaccaria F., "Physical Nonlinear Aspects of Classical and Quantum qOscillator",

Int. J. Mod. Phys. A, 8 (20), 121167, 1993.

[5] Man'ko V. I., Marmo G., Sudarshan E. C. G. and Zaccaria F.,

"f-Oscillators and Nonlinear Coherent States",

Phys. Scr., 55 (5), 528-541, 1997.

[6] Man'ko V. I. and Mendes R. V., "On the Nonlinearity Interpretation of q- and fDeformation and some Applications",

J. Phys. A: Math. Gen., 31 (28), 6037-6044, 1998.

[7] Gruver J. L.,

"q-Deformed Dynamics of q-Deformed Oscillators",

Phys. Lett. A, 254 (1-2), 1-6, 1999.

[8] Batouli J. and El Baz M.,

"Classical Interpretation of a Deformed Quantum Oscillator",

Found. Phys., 44 (2), 105-113, 2014.

[9] Lavagno A., Scarfone A. M., Swany P. N.,

"Classical and Quantum qDeformed Physical Systems",

Eur. Phys. J. C, 47 (1), 253-261, 2006.

[10] Eftekharzadeh A. and Hu B. L., "The Classical and Commutative Limit of Non-commutative Quantum Mechanics: A super * Wigner-Moyal Equation",

Braz. J. Phys., 35 (2A), 333-341, 2005. 
[11] Benatti F. and Gouba L.,

"Classical Limits of Quantum

Mechanics on a Non-

Commutative Configuration

Space",

J. Math. Phys., 54 (6), 2013.

[12] Benatti F. and Gouba L.,

"Interpretation of the Classical Limits of Quantum Mechanics on a Non-Commutative Configuration Space", ArXiv: $1409.5255 v 1$ [quantph] 18 Sep 2014 available at: http://arxiv.org/abs/1409.5255

[13] Zaslavskii O. B., Sinitsyn Y. A. and Tsukernik V. M.,

"Time Evolution of a Quantized Anharmonic Oscillator and Its Spin Analogue",

Sov. Phys. JETP, 64 (1), 90-95, 1986.

[14] Arik M. and Coon D. D.,

"Hilbert Space of Analytic Functions and Generalized Coherent States",

J. Math. Phys., 17 (4), 524-527, 1976.

[15] Biedenharn L.C.,

"The Quantum Group $\mathrm{SU}_{q}(2)$ and a q-Analogue of the Boson Operators",

J. Phys. A: Math. Gen., 22 (11), L873-L878, 1989.

[16] Macfarlane A.J.,

"On q-Analogues of the Quantum Harmonic Oscillator and the Quantum Group SU(2) $q$,

J. Phys. A: Math. Gen., 22 (1), 4581-4588, 1989.

[17] Quesne C.,

"New q-Deformed Coherent

States with an Explicitly Known Resolution of Unity",

J. Phys. A: Math. Gen., 35 (43) 9213-9226, 2002.
[18] Quesne C., Penson K. A. and Tkachuk V. M.,

"Maths. Type q-Deformed Coherent States for $q>1$ ",

Phys. Lett. A, 313 (1-2), 29-36 2003.

[19] Chaichian M. and Demichev A., "Introduction to Quantum Groups",

World Scientific Publishing Co. Pte. Ltd., Singapore, pp. 111-151, 1996.

[20] Isar A. and Scheid W.,

"Deformation of Quantum Oscillator and of its Interaction with Environment",

Physica A: Statistical Mechanics and its Applications, 335 (1-2), 79-93, 2004.

[21] Isar A.,

"Deformed Open quantum Systems",

Proceedings of the International Workshop, Bucharest, Romania, 7-12, Sep. 2003.

[22] Klauder J. R. and Sudarshan E. C. G.,

"Fundamentals of Quantum Optics",

W. A. BENJAMIN, INC., New York, pp. 105-135, 1968.

[23] Barnett S. M. and Radmore P. M., "Methods in Theoretical Quantum Optics",

Clarendon Press., Oxford, pp. 265-268, 1997.

[24] Puri R. R.,

"Mathematical Methods of Quantum Optics",

Springer, Berlin, pp. 48-50, 2001.

[25] Ahmed S. Mahmood and M. A. Z. Habeeb,

"The Classical Liouville Dynamics of the q-Deformed 1-D Classical Harmonic Oscillator",

Submitted for Publication in Journal of Al-Nahrain University, Science. 
[26] Ghosh G., Roy B. D. and Dey M., "On the Single-Particle Schrödinger Fluid",

J. Phys. G: Nucl. Phys., 3 (8), 1077-1084, 1977.

[27] Vitiello G.,

"Fractals, Coherent States and Self-Similarity Induced NonCommutative Geometry",

Phys. Lett. A, 376 (37), 25272532, 2012.

[28] Milburn G. J.,

"Quantum and Classical Liouville Dynamics of the Anharmonic Oscillator",

Phys. Rev. A, 33 (1), 674-685, 1986.

\section{الخلاصة}

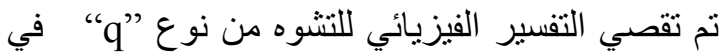

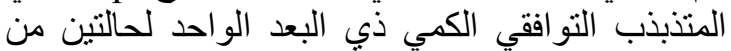

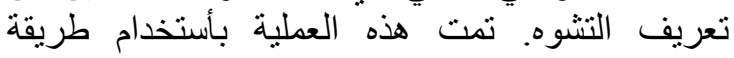

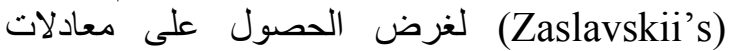
الحركة لهايزينبيرك (معادلات ليوفل الكمية) بدلالة

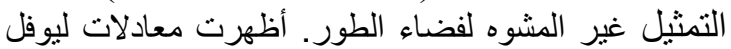

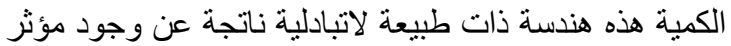

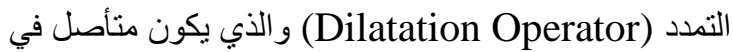

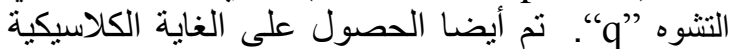
لمعادلات ليوفل الكمية بواسطة تطبيق شروط اليضي التئ حدودية

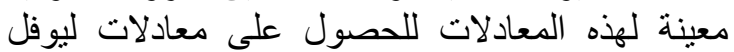

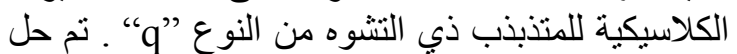

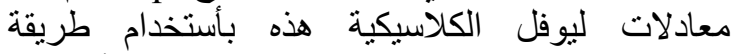
الخصائص للحصول على دوال توزيك لتوزيع الأحتمالية

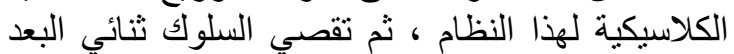

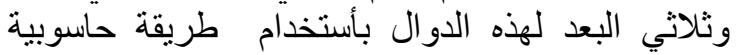

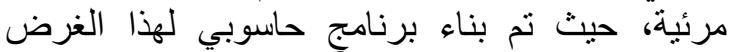

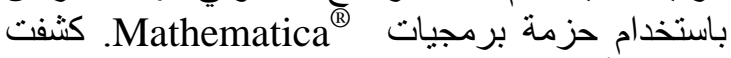
النتائج بأن الغاية الكلاسيكية لمعادلات ليوفل الكمية

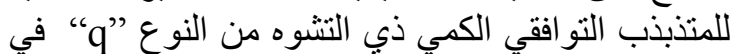

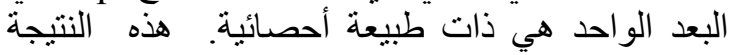
تلتوافق مع النتيجة التي حصل عليها (Ghosh et al. للمتذبذب التوافقي الكمي غير المشوه ذي البي البعد الواحد

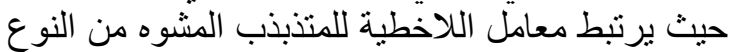

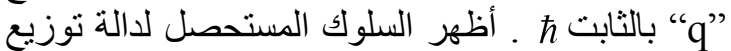
الأحتمالية الكلاسيكية اشكال لولبية تتطور مع الزمن في

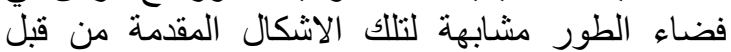
للمتذبذب اللاتو افقي الكلاسيكي ذي البعد (Milburn)

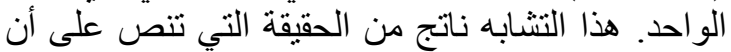

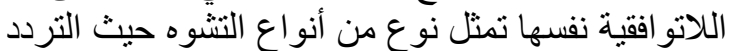
دالة للسعة. 